\title{
Aplasia and Hypoplasia of the Maxillary Sinus: Three Case Reports
}

\author{
Dmitrii A. Shcherbakov, $\mathrm{PhD}, \mathrm{ScD}^{1 *}$; Alexey V. Simonov ${ }^{2}$; Tatyana Yu. Malysheva ${ }^{3}$; \\ Ljalja A. Musina ${ }^{4}$; Aygul F. Shcherbakova ${ }^{1}$ \\ ${ }^{1}$ Tyumen State Medical University, Tyumen, Russia \\ ${ }^{2}$ Tyumen Morphology Center, Tyumen, Russia, ${ }^{3}$ Tyumen Regional Hospital, Tyumen, Russia \\ ${ }^{4}$ The Russian Eye and Plastic Surgery Center, Ufa, Russia
}

\begin{abstract}
Aplasia and hypoplasia of the maxillary sinus (MS) are developmental anomalies, the incidence ranging from 1.5\% to $10 \%$. Usually patients with aplasia and hypoplasia of MS are asymptomatic, but in some cases they complain of a runny nose, facial pain and pressure, and headache. These symptoms are very similar to maxillary sinusitis. Thus, it is important to distinguish anomalies from pathology. In the present study, three case reports illustrate the importance of correct diagnostics. (International Journal of Biomedicine. 2019;9(4):379-381.)
\end{abstract}

Key Words: maxillary sinus $\bullet$ developmental anomalies $\bullet$ aplasia $\bullet$ hypoplasia

\section{Introduction}

The maxillary sinus (MS) starts to develop in the 10th week of embryonic life. The volume of the sinus is about $3.3 \mathrm{~mm} \times 8.2 \mathrm{~mm} \times 2.8 \mathrm{~mm}$ at the time of birth. Then, up to 8 years, the MS increases annually by $2 \mathrm{~mm}$ in vertical and lateral dimensions and by $3 \mathrm{~mm}$ in the anteroposterior direction. At the age of 10-12 years, the inferior border of the sinus reaches the level of the nasal cavity floor. The growth of the MS lasts until age 12-20 years. ${ }^{(1)}$ The incidence of MS hypoplasia (MSH) ranges between $1.5 \%$ and $10 \% .{ }^{(2)}$ Usually, MSH is asymptomatic and is revealed by X-ray studies as an accidental find. ${ }^{(3)}$ But in some cases, ignorance of radiological signs of the MSH or use of conventional radiographs can lead to diagnostic problems, so MSH is identified as an infectious disease or cancer. ${ }^{(4)} \mathrm{Also}$, it is necessary to differentiate $\mathrm{MSH}$ from silent sinus syndrome. Thus, CT imaging and endoscopic examination are important diagnostic tools in cases of $\mathrm{MSH}$. Hereinafter, cases of MSH in real clinical practice are illustrated.

\section{Case Report 1}

A 26-year-old female was referred to the department of otorhinolaryngology at the medical-sanitary unit 'Neftyanik' with complaints of difficulty in nasal breathing, mucous discharge from the nasal cavity, and facial pain on the left side. The symptoms have bothered her for 3 years. X-ray of the nasal cavity and paranasal sinuses was repeatedly performed during exacerbations. Based on clinical and radiological findings, chronic maxillary sinusitis on the left side was diagnosed. During the exacerbation in 2018, a puncture of the left MS was performed, but no content was received. Medical therapy proved to be ineffective. Half a year after symptoms reappeared, a CT examination was performed and revealed MSH type 2 according to Bolger's classification, lateral position of the uncinate process, and type 3 right-side nasal septal deviation according to the classification of R. Mladina (Figure 1).

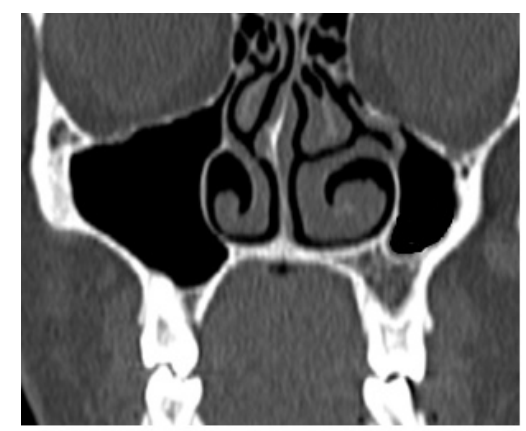

Fig. 1. Coronal CT scan. A decreased volume of the left MS, lateral position of the uncinate process-MSH type 2. 
The symptoms were due to the deviated nasal septum. Submucosal resection of nasal septum and correction of intranasal structures were performed. There were no symptoms after surgery, and no additional treatment was required. The patient was informed about the MSH.

\section{Case Report 2}

A 71-year-old female was admitted to the otorhinolaryngologist with complaints of facial pain on the left side, with a feeling of pressure in the left eye. Four years ago, the patient visited the otorhinolaryngologist with complaints of difficulty in nasal breathing, nasal discharge from the left half of the nasal cavity, and facial pain on the left side. CT was performed, the granuloma of root 6 of the upper tooth on the left was visualized. The patient was referred to the dentist-surgeon: the tooth was removed; the symptoms have disappeared. One year passed, the symptoms arose again and were not stopped by medical treatment. CT study was performed and evaluation of the coronal view revealed MSH type 2 according to Bolger's classification (Figure 2). The patient was referred to the department of otorhinolaryngology at the medical-sanitary unit 'Neftyanik' for surgery. An infundibulotomy was performed to access the MS; in the cavity of the MS a retention cyst was visualized and removed by using the shaver system. The postoperative course was uneventful, the patient was discharged in satisfactory health, and the symptoms were stopped.

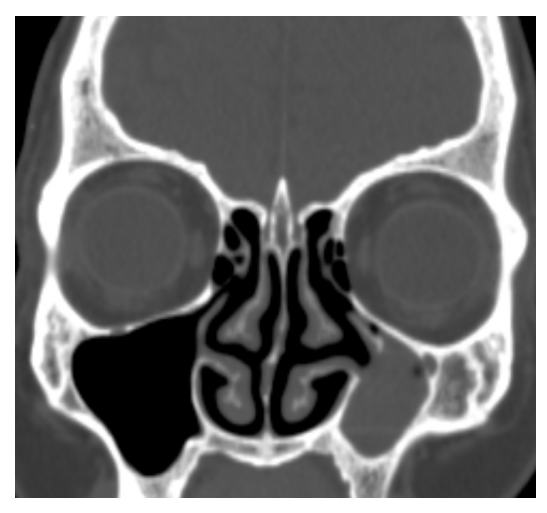

Fig. 2. Coronal CT scan.

Complete opacification and a smaller size of the left MS, lateral position of the uncinate process - MSH type 2.

\section{Case Report 3}

A 36-year-old man was referred to the department of otorhinolaryngology at the medical-sanitary unit 'Neftyanik' with complaints of nasal discharges from the right half of the nasal cavity, lasting for 4 months. Before that, a puncture of the right MS had been performed twice, without giving relief. The patient was referred to CT examination, which showed a decreased volume of both MSs, opacification of the right MS, lateralized uncinate process, and fontanelle, which corresponds to type 2 hypoplasia according to Bolger's classification (Figure 3).

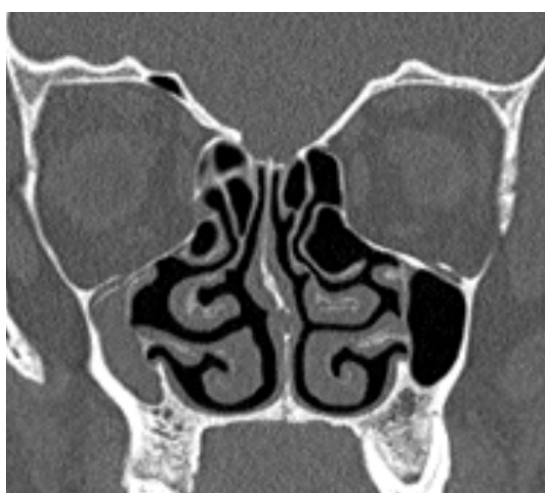

Fig. 3. Coronal CT scan. A decreased volume of both MSs, the bilateral uncinate process-MSH type 2.

The right uncinate process was resectioned, the ostium of the right MS was enlarged, and sinus contents were removed. The postoperative course was uneventful; the patient was discharged in satisfactory health. During a followup examination after 6 months there were no complaints.

\section{Discussion}

The clinical cases given above demonstrate the need for high caution regarding the abnormalities of the MS. MSH is a rare condition and it is usually asymptotic. However, in some cases, there exist some nasal symptoms, which are associated with a deviated nasal septum or lateralized uncinate process. In this state of health, the MS drainage is impaired and surgery is required. MSH may lead to diagnostic problems. Usually it is disguised as chronic rhinosinusitis. ${ }^{(5)}$ In these cases, medical therapy has no effect, and an attempt to puncture of the MS can lead to injury of the orbit. ${ }^{(6,7)}$ Thus, CT imaging is the necessary tool to diagnose MSH. CT images characteristic of MSH are the impression of anterior and lateral walls into the sinus cavity and a decrease in horizontal and vertical dimensions, the maximum values of which are less than $50 \%$ of the diameter of the orbit. ${ }^{(8)}$ It is very important to distinguish MSH from silent sinus syndrome, which always leads to enophthalmos. ${ }^{(9)}$ The abnormal type of the MS is associated with abnormalities of the uncinate process and infundibulum. According to Bolger's classification, in MSH type 1 a mild hypoplasia is combined with normally developed uncinate process and with well-developed infundibulum. In MSH type 2 , significant hypoplasia is associated with hypoplastic/absent uncinate process and poorly defined or absent infundibulum. The MSH type 3 is characterized as profound hypoplasia with absent uncinate process. ${ }^{(10)}$ Thus, it is necessary to identity abnormalities of the MS. When MSH is diagnosed in time, it can save the patient from long-term medical treatment with no effect, performing unnecessary puncture of the MS, injury to the orbit, and just wasting of time. The main treatment of MSH in cases of the given symptoms is an endoscopic surgery.

*Corresponding author: Prof. Dmitrii A. Shcherbakov, PhD, ScD. Tyumen State Medical University, Tyumen, Russia. E-mail: dmst@,bk.ru 


\section{Competing Interests}

The authors declare that they have no competing interests.

\section{References}

1. Kosko JR, Hall BE, Tunkel DE. Acquired maxillary sinus hypoplasia: A consequence of endoscopic sinus surgery? Laryngoscope. 1996;106(10):1210-3.

2. Thiagarajan B, Narashiman NS. Hypoplasia of all paranasal sinuses: A case series and literature review. Otolaryngology Online Journal. 2012; 2(2);125-128.

3. Hupp JR, Ellis E, Tucker MR. Contemporary Oral and Maxillofacial Surgery. 5th ed. St. Louis: Mosby Elsevier; 2008.

4. Baykara M, Erdouan N, Ozturk M, Erkan M. Maxillary sinus aplasia. Turk J Med Sci. 2002;32:273-275.

5. Pino Rivero V, Pardo Romero G, Rodríguez Carmona M, Iglesias González RJ, del Castillo Beneyto F. [Maxillary sinus hypoplasia masquerading a chronic sinusitis]. An
Otorrinolaringol Ibero Am. 2007;34(4):323-8. [Article in Spanish].

6. Tasar M, Cankal F, Bozlar U, Hidir Y, Saglam M, Ors F. Bilateral maxillary sinus hypoplasia and aplasia: radiological and clinical findings. Dentomaxillofac Radiol. 2007;36(7):412-5.

7. Wake M, Shankar L, Hawke M, Takeno S. Maxillary sinus hypoplasia, embryology, and radiology. Arch Otolaryngol Head Neck Surg. 1993;119(12):1353-7.

8. Sirikçi A, Bayazit Y, Gümüsburun E, Bayram M, Kanlikana M. A new approach to the classification of maxillary sinus hypoplasia with relevant clinical implications. Surg Radiol Anat. 2000;22(5-6):243-7.

9. Bierny JP, Dryden R. Orbital enlargement secondary to paranasal sinus hypoplasia. AJR Am J Roentgenol. 1997;128(5):850-2.

10. Bolger WE, Woodruff WW Jr, Morehead J, Parsons DS. Maxillary sinus hypoplasia: classification and description of associated uncinate process hypoplasia. Otolaryngol Head Neck Surg. 1990;103(5 ( Pt 1)):759-65. 